\title{
Filtering Atmost1 on Pairs of Set Variables
}

\author{
Willem-Jan van Hoeve ${ }^{1}$ and Ashish Sabharwal ${ }^{2 \star}$ \\ 1 Tepper School of Business, Carnegie Mellon University \\ 2 Department of Computer Science, Cornell University
}

\section{Introduction}

Many combinatorial problems, such as bin packing, set covering, and combinatorial design, can be conveniently expressed using set variables and constraints over these variables [3]. In constraint programming such problems can be modeled directly in their natural form by means of set variables. This offers a great potential in exploiting the structure captured by set variables during the solution process, for example to break problem symmetry or to improve domain filtering.

We present an efficient filtering algorithm, establishing bounds consistency, for the atmost 1 constraint on pairs of set variables with fixed cardinality. Computational results on social golfer benchmark problems demonstrate that with this additional filtering, these problems can be solved up to 50 times faster.

\section{Domain Filtering for Set Constraints}

A set variable is a variable whose domain values are sets. As the number of possible values of a set variable can be enormous (the size of a power set, in the worst case), one usually represents the domain of a set variable $S$ by an interval $[L(S), U(S)]$, where $L(S)$ and $U(S)$ are a 'lower' and 'upper' bound on the values that $S$ can take. In addition, a lower bound $l(S)$ and upper bound $u(S)$ on the cardinality of $S$ are maintained. A natural (and widely adopted) representation for the domain of set variables is based on the subset ordering of the domain. That is, the lower bound $L(S)$ represents all mandatory elements, while the upper bound $U(S)$ represents all possible elements, i.e., $D(S)=\{s \mid$ $L(S) \subseteq s \subseteq U(S), l(S) \leq|S| \leq u(S)\}$. We refer to this representation as the subset+cardinality representation. It is applied in CP solvers such as ILOG Solver, Eclipse, and Gecode.

For constraints involving set variables, the filtering task is to increase the lower bounds and decrease the upper bounds of the domains such that we achieve bounds consistency, which should formally be called subset+cardinality-bounds consistency in our case:

Definition 1. Let $S_{1}, \ldots, S_{n}$ be set variables. A constraint $C\left(S_{1}, \ldots, S_{n}\right)$ is called subset+cardinality-bounds consistent if for all $i=1, \ldots, n, L\left(S_{i}\right)$ and

\footnotetext{
* This research was partly supported by the Intelligent Information Systems Institute, Cornell University under AFOSR Grant FA-9550-04-1-0151.
} 
$U\left(S_{i}\right)$ are the intersection and the union, respectively, of all values in $D\left(S_{i}\right)$ that can be assigned to $S_{i}$ in a solution to $C$, while in addition $l\left(S_{i}\right)$ and $u\left(S_{i}\right)$ are equal to the minimum and maximum cardinality over these values, respectively.

When a filtering algorithm for set constraints does not necessarily establish bounds consistency, we call it a partial filtering algorithm.

\section{The Atmost1 Constraint on Pairs of Set Variables}

The atmost 1 constraint was introduced by Sadler and Gervet [5] and specifies, for a collection of $n$ set variables with given cardinalities, that each pair of variables overlaps in at most one element. Filtering the atmost 1 constraint to bounds consistency is NP-hard [1]. Therefore, Sadler and Gervet [5] give in on bounds consistency and present a partial filtering algorithm. In this work, we given in on the number of variables instead, and consider the atmost 1 constraint involving two set variables only, which we will refer to as the pair-atmost1 constraint. Formally, pair-atmost $1\left(S_{1}, S_{2}, c_{1}, c_{2}\right)=\left\{\left(s_{1}, s_{2}\right) \mid s_{1} \in D\left(S_{1}\right), s_{2} \in\right.$ $\left.D\left(S_{2}\right),\left|s_{1}\right|=c_{1},\left|s_{2}\right|=c_{2},\left|s_{1} \cap s_{2}\right| \leq 1\right\}$, where $S_{1}$ and $S_{2}$ are set variables and $c_{1}, c_{2} \geq 1$ are integers representing the cardinalities of $S_{1}$ and $S_{2}$, respectively.

A natural way of implementing the pair-atmost1 constraint is to use the following decomposition of pair-atmost $1\left(S_{1}, S_{2}, c_{1}, c_{2}\right)$ into three constraints: $\left|S_{1}\right|=c_{1}, \quad\left|S_{2}\right|=c_{2}, \quad\left|S_{1} \cap S_{2}\right| \leq 1$. We will refer to this as the decomposition for pair-atmost1. Unfortunately, filtering these constraints separately does not establish bounds consistency on the pair-atmost1 constraint, as illustrated by the following example:

Example 1. Let $D\left(S_{1}\right)=[\{1,2\},\{1,2,3,5,6\}], D\left(S_{2}\right)=[\{3\},\{1,2,3,4\}]$, and $c_{1}=c_{2}=3$. Establishing bounds consistency on pair-atmost $1\left(S_{1}, S_{2}, c_{1}, c_{2}\right)$ leads to $D\left(S_{1}\right)=[\{1,2\},\{1,2,5,6\}], D\left(S_{2}\right)=[\{3,4\},\{1,2,3,4\}]$. This will not be achieved by the decomposition.

\section{The Bounds Consistency Filtering Algorithm}

We next present the filtering algorithm that establishes bounds consistency on the pair-atmost1 constraint, which we call BC-FilterPairAtmost1 (shown as Algorithm 1).

First, we partition each of $D\left(S_{1}\right)$ and $D\left(S_{2}\right)$ into six disjoint sets. For this purpose we define $\mathrm{L} 1=L\left(S_{1}\right)$ and $\mathrm{P} 1=U\left(S_{1}\right) \backslash L\left(S_{1}\right)$, i.e., L1 represents the lower bound, and P1 the possible values, for $S_{1}$. We define L2 and P2 similarly for $D\left(S_{2}\right)$. Using these shorthands, we define the partition of $D\left(S_{1}\right)$ into L1only $=L 1 \backslash U\left(S_{2}\right), \mathrm{L} 1 \mathrm{~L} 2=L 1 \cap L 2, \mathrm{~L} 1 \mathrm{P} 2=L 1 \cap P 2, \mathrm{P} 1 \mathrm{~L} 2=P 1 \cap L 2, \mathrm{P} 1 \mathrm{P} 2$ $=P 1 \cap P 2$, and P1only $=P 1 \backslash U\left(S_{2}\right) . D\left(S_{2}\right)$ is similarly partitioned into L2only, L2L1, L2P1, P2L1, P2P1, and P2only. Note that L1L2 = L2L1, P1L2 = L2P1, and P2L1 = L1P2. For these three pairs, we explicitly maintain only one set per pair, namely, L1L2, P1L2, and P2L1, respectively. (While P1P2 = P2P1 as well, we still need to maintain both of these sets.) 


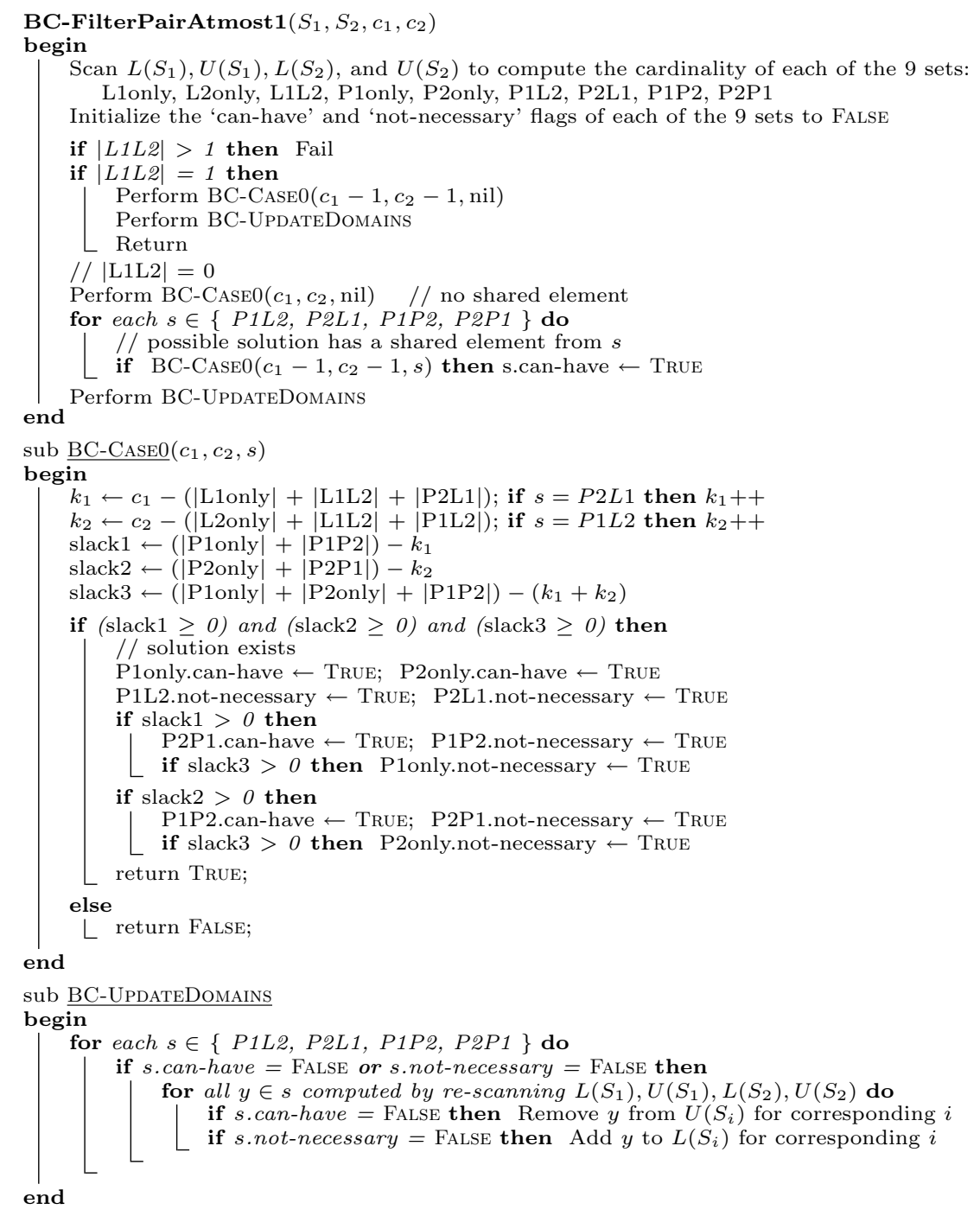

Algorithm 1: Bounds consistency domain filtering for pair-atmost1.

Example 2. For the scenario of Example 1, we have $\mathrm{L} 1=\{1,2\}, \mathrm{P} 1=\{3,5,6\}$, $\mathrm{L} 2=\{3\}$, and $\mathrm{P} 2=\{1,2,4\}$. The 9 sets in this case are: L1only $=\varnothing$, L2only $=\varnothing, \mathrm{L} 1 \mathrm{~L} 2=\varnothing, \mathrm{P} 1$ only $=\{5,6\}, \mathrm{P} 2$ only $=\{4\}, \mathrm{P} 1 \mathrm{~L} 2=\{3\}, \mathrm{P} 2 \mathrm{~L} 1=\{1,2\}$, $\mathrm{P} 1 \mathrm{P} 2=\varnothing$, and $\mathrm{P} 2 \mathrm{P} 1=\varnothing$.

For each of the 9 sets, we maintain two Boolean flags: The "can-have" flag and the "not-necessary" flag, that are all initialized to FALSE. Some of them will be set to True during the course of the algorithm when we find a solution. If at the end, for a set $s, s$.can-have is still FALSE, we remove $s$ from the upper 
bound of the corresponding domain. If $s$.not-necessary is still FALSE, we add $s$ to the lower bound.

We find a solution by comparing the cardinalities of the 9 sets. In our base case (BC-CASE0), we assume that the variables already have one element in common. For $S_{1}$ we need $k_{1}=c_{1}-\left|L\left(S_{1}\right)\right|-1$ additional values (or one more, if the common element was in $L\left(S_{1}\right)$ ). Similarly, we need $k_{2}$ more values for $S_{2}$. If we can meet the demand (verified by nonnegativity of slack1, slack2, and slack3 Algorithm 1), there exists a solution, and we update the flags for our 9 sets.

When we are not in the base case, i.e., L1L2 $=0$, there are two possibilities. First, there could be a solution in which there is no common element. For this we run the base case, as is. Second, there will be a shared element, originating from P1L2, P2L1, P1P2, or P2P1. For each of these possibilities, we 'remove' the shared element from $S_{1}$ and $S_{2}$, which brings us in the base case again.

Theorem 1. Algorithm 1 establishes bounds consistency on the pair-atmost1 constraint.

Theorem 1 can be proved by a careful case analysis. The time complexity of BC-FilterPairAtmost 1 is dominated entirely by the creation of the 9 sets during search, which takes $O(n)$ time where $n$ is the integer domain size. The rest of the algorithm has only a constant number of calls to BC-CASE0 and one call to BC-UpdateDomains. BC-UpdateDomains takes time $O(n+k \log n)$, where $k$ is the number of elements removed from an upper bound or added to a lower bound, assuming standard set operations used for maintaining these upper and lower bounds take time $O(\log n)$. We can tighten this analysis by amortizing over an entire path in the search tree from the root to any leaf, such that the total filtering complexity is $O(n \log n)$, while updating the flags takes total time $O(n)$, for the path.

\section{Experimental Results}

We evaluated the performance of the pair-atmost1 constraint on the well-known social golfer problem (problem prob010 in CSPLib). The problem golf $-g-s-w$ asks for a partition of $n$ golfers into $g$ groups, each of size $s$, for $w$ weeks, such that no two golfers are in the same group more than once throughout the whole schedule. We apply the following standard model, using set variables $S_{i j}$ to represent the set of golfers of week $i$ and group $j$ :

$$
\begin{array}{ll}
\text { partition }\left(S_{i 1}, \ldots, S_{i g},\{1, \ldots, n\}\right), & 1 \leq i \leq w \\
\text { pair-atmost } 1\left(S_{i j}, S_{k l}, s, s\right), & 1 \leq i<k \leq w, 1 \leq j \leq g, 1 \leq l \leq g \\
\left|S_{i j}\right|=s, & 1 \leq i \leq w, 1 \leq j \leq g \\
S_{i j} \in[\varnothing,\{1, \ldots, n\}], & 1 \leq i \leq w, 1 \leq j \leq g .
\end{array}
$$

To speed up the computation, we also applied a redundant global cardinality constraint [4] on integer variables $x_{i j}$ representing the group in which golfer $j$ plays in week $i$. Our search strategy is a smallest-domain-first on these variables. 


\begin{tabular}{|c|c|c|c|c|}
\hline \multirow[t]{2}{*}{ Problem } & \multicolumn{2}{|c|}{$\begin{array}{l}\text { Decomposition } \\
\text { (partial filtering) }\end{array}$} & \multicolumn{2}{|c|}{$\begin{array}{l}\text { BC-FilterPair Atmost1 } \\
\text { (bounds consistency) }\end{array}$} \\
\hline & time (s) & backtracks & time (s) & backtracks \\
\hline golf-6-5-5 & 2106.7 & $10,986,224$ & 75.5 & 239,966 \\
\hline golf $-6-5-4$ & 1517.7 & $10,930,370$ & 39.7 & 197,837 \\
\hline golf $-6-5-3$ & 1060.5 & $10,930,016$ & 29.6 & 197,607 \\
\hline golf $-6-5-2$ & 635.5 & $10,879,368$ & 17.2 & 171,664 \\
\hline golf $-8-4-4$ & 226.7 & $1,555,561$ & 157.7 & 738,393 \\
\hline golf-10-3-10 & 128.1 & 150,911 & 67.2 & 78,976 \\
\hline golf-10-3-9 & 86.0 & 150,452 & 52.4 & 78,613 \\
\hline golf-10-3-6 & 21.3 & 110,429 & 17.3 & 57,364 \\
\hline golf-10-4-5 & 51.3 & 310,110 & 4.5 & 22,044 \\
\hline golf-10-4-4 & 42.5 & 310,109 & 4.0 & 22,043 \\
\hline golf $-7-4-4$ & 22.5 & 184,641 & 4.4 & 27,877 \\
\hline
\end{tabular}

Table 1. Computational results on a number of social golfer instances.

Finally, to account for some symmetry-breaking, we partly instantiate some of the set variables before starting the search, following Fahle et al. [2]. We note that our filtering algorithm can be applied to any model, including those with more advanced symmetry-breaking techniques.

We implemented our model in ILOG Solver 6.3, and all experiments run on a $3.8 \mathrm{GHz}$ Intel Xeon machine with 2 GB memory running Linux 2.6.922.ELsmp. We evaluated the performance of the decomposition implementation of pair-atmost1 (achieving partial filtering) with our filtering algorithm BCFiLTERPAirAtMost1 (achieving bounds consistency) on a number of instances, as reported in Table 1. The results demonstrate that using the bounds consistency algorithm, one can solve these instances up to 50 times faster, with a similar reduction in the number of search tree backtracks.

\section{References}

[1] C. Bessiere, E. Hebrard, B. Hnich, and T. Walsh. Disjoint, partition and intersection constraints for set and multiset variables. In $C P^{\prime} 04$, volume 3258 of $L N C S$, pages 138-152, 2004.

[2] T. Fahle, S. Schamberger, and M. Sellmann. Symmetry breaking. In $C P^{\prime}$ 01, volume 2239 of $L N C S$, pages 93-107, 2001.

[3] C. Gervet. Constraints over structured domains. In F. Rossi, P. van Beek, and T. Walsh, editors, Handbook of Constraint Programming. Elsevier, 2006.

[4] J.-C. Régin. Generalized arc consistency for global cardinality constraint. In AAAI'96, volume 1, pages 209-215, 1996.

[5] A. Sadler and C. Gervet. Global reasoning on sets. In Proc. of Workshop on Modelling and Problem Formulation (FORMUL'01), 2001. 